\title{
INSTRUMENTOS DE EVALUACIÓN DE LA CULTURA EMPRENDEDORA
}

\author{
María Eugenia Martin Palacio \\ Universidad Complutense de Madrid \\ mariaeugeniamartin@edu.ucm.es \\ Francis Edie Flores Castillo \\ Universidad de Playa Ancha \\ francisedie@gmail.com \\ Cristina di Giusto Valle \\ Universidad de Burgos \\ cristinadgv@gmail.com
}

Fecha de Recepción: 27 Febrero 2019

Fecha de Admisión: 30 Abril 2019

\section{RESUMEN}

Se presenta el proceso de construcción y resultados de cuatro instrumentos que reúnen requisitos psicométricos de fiabilidad, validez y normalización y evalúan la cultura emprendedora (perciba y deseada) en contextos de educación secundaria en relación a sus cuatro componentes: la finalidad de la educación, procesos de enseñanza, procesos de aprendizaje y escenario educativo, en alumnos y profesores.

La muestra estuvo conformada en la fase piloto por 173 alumnos de dos centros educativos públicos (27.7\% y el $72.3 \%$ respectivamente). El 52.6\% eran hombres. En relación al curso, la

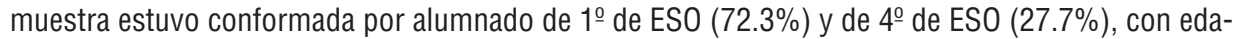
des comprendidas entre los 11 y los 17 años, siendo la media 12.99 y la desviación típica 1.73. La muestra del profesorado estuvo formada por 63 participantes de tres centros educativos públicos (42.9\%, $42.9 \%$ y $14.3 \%$ respectivamente). De los docentes, el $33.3 \%$ eran hombres y el $66.7 \%$ eran mujeres, con edades comprendidas entre los 31 y los 68, siendo la media 50.49 y la desviación típica 6.79 .

La muestra empírica ha estado formada por 2308 discentes (47.7\% hombres) de 18 centros educativos, tanto públicos como concertados y privados. La muestra de discentes estuvo formada por estudiantes desde $1^{\circ}$ ESO hasta $2^{\circ}$ Bachillerato, con una media de edad de 14.84 y una desviación típica de 1.77. La muestra del profesorado se compuso de 176 (32.4\% hombres) participantes de 16 centros tanto públicos como concertados. La media de edad fue 47.92, con una desviación típica de 7.74. Se agrupó a los docentes en ramas educativas: $32.4 \%$ de Ciencias; $66.5 \%$ de Letras y $3 \%$ de Arte. 


\section{INSTRUMENTOS DE EVALUACIÓN DE LA CULTURA EMPRENDEDORA}

Los resultados obtenidos de fiabilidad, validez factorial y confirmatoria y baremos permiten con las garantías psicométricas pertinentes su aplicación tanto en profesores como en alumnos en contextos de educación secundaria.

Palabras clave: evaluación; cultura; emprendimiento

\section{ABSTRACT}

Instruments of evaluation of the enterprising culture. It presenst the process of construction and results of four instruments that meet psychometric requirements of reliability, validity and normalization and evaluate the entrepreneurial culture (perceive and desired) in secondary education contexts in relation to their Four components: The purpose of education, teaching processes, learning processes and educational scenario, in students and teachers.

The sample was formed in the pilot phase by 173 students from two public schools $(27.7 \%$ and $72.3 \%$ respectively). $52.6 \%$ were men. In relation to the course, the sample was formed by students of $1^{\circ}$ of $\operatorname{ESO}(72.3 \%)$ and of $4 \stackrel{\circ}{\circ}$ of ESO $(27.7 \%)$, with ages between 11 and 17 years, being the mean 12.99 and the typical deviation 1.73. The teaching staff sample consisted of 63 participants from three public schools $(42.9 \%, 42.9 \%$ and $14.3 \%$ respectively). Of the teachers, $33.3 \%$ were men and $66.7 \%$ were women, aged between 31 and 68, with the mean 50.49 and the typical deviation 6.79 .

The Empirical sample has been formed by 2308 Dicentes ( $47.7 \%$ men) of 18 educational centers, both public and concerted and private. The Dicentes sample consisted of students from 1st to $2 \stackrel{\circ}{ }$ High, with an average age of 14.84 and a typical deviation of 1.77 . The Teacher's sample was composed of 176 (32.4\% men) participants from 16 public and concerted centres. The mean age was 47.92 , with a typical deviation of 7.74 . Teachers Are grouped in educational branches: $32.4 \%$ of Science; $66.5 \%$ of Letters and 3\% of Art.

The results obtained of reliability, factorial validity and confirming and bares allow with the pertinent psychometric guarantees their application both in professors and in students in contexts of secondary education.

Keywords: evaluation; culture; entrepreneurship

\section{INTRODUCCIÓN}

Existen diferentes instrumentos que permiten evaluar la cultura escolar. El problema radica en la elección del modelo que permita su análisis en las mejores condiciones de comprensibilidad, operatividad y uso acorde con las líneas de investigación de los paradigmas existentes considerados más actuales en el campo de la educación.

Meza (2010) señala que durante los años 80 la mayoría de las investigaciones sobre la cultura escolar se basaron en estudios cualitativos en un número reducido de centros educativos. En la década de los 90 se elaboraron instrumentos para llevar a cabo las evaluaciones a un número más amplio de centros, construyéndose varios cuestionarios como los de Pang (1995); Grady, Fisher y Fraser (1996); Houtveen, Voogt, Van der Vegt y Van de Grift (1996); o Jones (1996).

Marcone y Martín del Buey (2003), además de elaborar y presentar el Inventario de Cultura Organizacional Educativa (ICOE), también realizaron la siguiente recopilación de cuestionarios existentes: Scaq School Culture Assessment Questionnaire (Sashkin y Sashkin, 1990); Inventario de Cultura Organizacional Innovadora (Reyes y Zambrano, 1991); Diagnóstico de la Cultura Organizacional (Harrison y Stokes, 1992); Cuestionario de Cultura Organizacional (García, 1994) ; Organizational Culture Survey (Denison y Neale, 1994, 2000). Se realizó una adaptación al castellano de este cuestionario en el 2009 (Bonavia, Prado y Barberá, 2009). 
No obstante, siguiendo la línea por la que se ha optado en esta investigación en torno al desarrollo de la cultura de emprendimiento tan necesaria en contextos educativos universitarios (Sánchez, Caggiano, y Hernández, B. (2011), De la Torre, Ruiz, E. Escolar, Baños, Gañan Corbi, Santos y Sánchez, I (2015), Luis, Palmero, De la Torre, Ruiz, Corbi , Sánchez, y Jiménez (2015) parece pertinente optar por la construcción de cuestionarios afines al modelo de análisis propuesto en el constructo Cultuaula formulado por Martín del Buey y Martín Palacio (Martín del Buey, 1991 Martín del Buey y Martín Palacio (2012); y desarrollado en diferentes trabajos en los últimos años Arnaiz, Pizarro y Martín del Buey, 2012; Pizarro, Arnaiz y Dapelo (2012);Martín Palacio, Aguado y Rodríguez (2013); Arnaiz, Di Giusto y Zamorano, 2014; y Di Giusto, Castellanos y Arnaiz, 2014). Y de manera destacada en la tesis doctoral de Arnaiz, 2015).

El modelo considera que la cultura es el conjunto de creencias, valores y normas compartidas por una comunidad. No obstante, se considera que el núcleo o la base de la cultura son las creencias. Son éstas las que se van a someter a evaluación en esta investigación. En el modelo las creencias se diferencian en cuatro tipos: Finalidad educativa; Proceso de enseñanza; Proceso de aprendizaje y Escenario educativo.

A este respecto es oportuno señalar la existencia de un breve cuestionario denominado Cultuaula formulado por Martín del Buey (1997) compuesto de 20 ítems, distribuidos en 4 bloques relacionados con la finalidad $(\mathrm{F})$, proceso de instrucción $(\mathrm{PI})$, proceso de aprendizaje $(\mathrm{PA})$ y escenario educativo.

Sobre ese cuestionario base se han venido haciendo adaptaciones aplicables a distintos niveles educativos. La adaptación realizada del cuestionario a niveles de educación secundaria realizada por Antúnez (2012), representa un buen intento, aunque con algunas carencias que no permiten su utilización dado que no reúne la totalidad de los requisitos psicométricos de todo instrumento psicológico.

En este sentido se impone la necesidad de construir un cuestionario acorde con el modelo propuesto que reúna todas las características de fiabilidad, validez de estructura, de contenido y los baremos correspondientes con el objetivo de poder valorar con las máximas garantías la presencia 0 ausencia de la cultura emprendedora en contextos de enseñanza secundaria.

\section{METODOLOGíA}

Previo a su fase empírica se realizó un estudio piloto.

El estudio piloto cualitativo se realizó con 5 docentes y 16 alumnos, de diferentes cursos de Educación Secundaria. Se les pidió que evaluaran la claridad de los ítems con el objetivo de comprobar si entendían lo que se quería evaluar con los mismos. A continuación, se llevó a cabo un muestreo no probabilístico de carácter intencional, con alumnado y profesorado voluntario.

La muestra de los estudiantes estuvo formada por 173 participantes de dos centros educativos públicos. El 52.6\% eran hombres y el $47.4 \%$ mujeres. En relación al curso, la muestra estuvo conformada por alumnado de $1^{\circ}$ de ESO $(72.3 \%)$ y de $4^{\circ}$ de ESO $(27.7 \%)$, con edades comprendidas entre los 11 y los 17 años.

La muestra del profesorado estuvo formada por 63 participantes de tres centros educativos públicos (42.9\%, 42.9\% y $14.3 \%$ respectivamente); el $33.3 \%$ eran hombres y el $66.7 \%$ eran mujeres, con edades comprendidas entre los 31 y los 68 .

Para el análisis de los datos se empleó el paquete estadístico IBM SPSS Statistics 18.0 y el programa Factor 9.2 (Lorenzo-Seva y Ferrando, 2013).

Se llevaron a cabo los análisis de fiabilidad, mediante el índice de consistencia interna coeficiente Alfa de Cronbach (Cronbach, 1951); y se realizaron Análisis Factoriales Exploratorios (AFE) para cada uno de los cuestionarios. 


\section{INSTRUMENTOS DE EVALUACIÓN DE LA CULTURA EMPRENDEDORA}

El alfa de Cronbach del cuestionario Cultura emprendedora percibida alumnado es de .952. El de deseado alumnado es de 940 . El de Cultura emprendedora percibida profesorado es de .853 y el Cultura emprendedora deseada profesorado es de .951.

A través del programa FACTOR 9.2, se realiza un análisis factorial de naturaleza exploratoria de la estructura factorial de los cuestionarios.

El resultado final fue:

24 ítems, que medían cuatro factores:

- Finalidad educativa: ítems 1, 4, 9, 14, 17 y 22

- Proceso de enseñanza: ítems 2, 5, 10, 12, 18 y 19

- Proceso de aprendizaje: ítems 3, 7, 13, 15, 21 y 23

- Escenario: ítems 6, 8, 11, 16, 20 y 24.

El formato de respuesta de los cuestionarios se presenta en escala Likert, a través de la cual se ofrece una afirmación al sujeto y se le pide que la califique de 1 a 5 , según el grado de acuerdo con la misma, donde 1 es igual a "nunca" y 5 es equivalente a "siempre".

Finalizada esta parte se procedió a la aplicación empírica del instrumento con la finalidad de obtener los índices de fiabilidad, validez y normalización pertinentes.

\section{Participantes}

La muestra de estudiantes estuvo formada por 2308 participantes de 18 centros educativos. El $73.6 \%$ de los alumnos (1698) pertenecían a institutos públicos, el 23.5\% (542) pertenecían a centros concertados, y el 2.9\% (68 se encontraban en centros privados. Los estudiantes participantes de este estudio eran 1101 hombres (47.7\%) y 1203 mujeres (52.1\%), encontrándose 4 personas que no indicaron su género $(0.2 \%)$, La frecuencia del curso del alumnado, donde se muestra que el $16.5 \%$ era de $1^{\circ}$ ESO; el $14.4 \%$ de $2^{\circ}$ ESO; el $13 \%$ de $3^{\circ}$ ESO; el $20.4 \%$ de $4^{\circ}$ ESO; $21.9 \%$ de $1^{\circ}$ Bachillerato; y el $13.7 \%$ de 20 Bachillerato. La edad del alumnado comprende desde los 10 hasta los 27 años, siendo la media 14.84 y la desviación típica 1.77. En cuanto a la procedencia del alumnado, la muestra estuvo formada por 1922 estudiantes de Asturias (83.3\%), 372 de Castilla y León $(16.1 \%)$ y 14 de Cantabria $(0.6 \%)$,

La muestra de profesorado estuvo formada por 176 participantes de 16 centros educativos. Según el tipo de centro había 133 docentes de centros públicos (75.6\%) y 43 de centros concertados (24.4\%). Los centros de educación privada no aparecen reflejados debido a que se trata de colegios concertados donde los cursos de Bachillerato son privados, pero el personal docente es el mismo en Educación Secundaria Obligatoria y en Bachillerato, por lo que se les considera a todos como pertenecientes a centros concertados. En relación al género, los docentes que participaron en este estudio fueron en su gran mayoría mujeres; $66.5 \%$ profesoras frente al $32.4 \%$ de los profesores. La edad del profesorado, comprende desde los 28 hasta los 64 años, siendo la media 47.92 y la desviación típica 7.47. La edad de los docentes, al tener una amplia variedad, se dividió en tres grupos: hasta los 40 años; entre los 41 y 50 años; y de los 51 años en adelante. El primer grupo supone el $18.8 \%$ de los participantes; el segundo grupo supone $33.5 \%$; y el tercer grupo el $36.4 \%$, encontrándose 20 docentes que no indicaron su edad. El tipo de asignaturas que imparten los docentes, agrupándolas por ramas educativas: Ciencias (32.4\%), Letras (66.5\%) y Arte (3\%). La rama de Ciencias incluye asignaturas como Física y Química; Biología; Matemáticas; etc. La rama de Letras incluye a asignaturas como Lengua y Literatura; Historia de España; Inglés; Francés; etc. Por último, la rama de Arte recoge asignaturas como Música o Educación Plástica.

El profesorado del presente estudio impartía clases en Educación Secundaria Obligatoria, en Bachillerato 0 en ambos cursos. la muestra estuvo formada por 133 docentes de Asturias (75.6\%), 42 de Castilla y León (23.9\%) y uno de Cantabria (0.6\%). 


\section{Procedimiento}

La aplicación de los cuestionarios al alumnado tuvo lugar en el aula habitual dentro del horario escolar. El centro educativo ofreció una hora lectiva o de tutoría para realizar la aplicación de la prueba en formato de lápiz y papel. La aplicación se realizó de forma colectiva y en una única sesión, sin establecer limitación temporal a los participantes, oscilando el tiempo de respuesta a los dos cuestionarios entre los 15 y 20 minutos aproximadamente.

La aplicación de los instrumentos para el profesorado se realizó de dos formas posibles: En la sesión en la que el alumnado cumplimentaba sus instrumentos correspondientes. En la mayoría de Ios casos, si la aplicación al alumnado tenía lugar en hora de tutoría, el tutor/a de cada grupo cubría sus cuestionarios en esa misma hora.

En la sala de profesores. El motivo de ofrecer dos contextos de aplicación en el caso del profesorado es porque de este modo se podía facilitar que un mayor número de docentes cumplimentaran los instrumentos.

\section{Análisis de los datos}

Para el análisis de los datos del estudio se empleó el paquete estadístico IBM SPSS Statistics 18.0, el AMOS 18, y el programa Factor 9.3.1 (Lorenzo-Seva y Ferrando, 2015).

Para estudiar las características psicométricas de los cuatro instrumentos se llevaron a cabo los análisis de fiabilidad, mediante el índice de consistencia interna coeficiente Alfa de Cronbach (Cronbach, 1951). Asimismo se realizaron Análisis Factoriales Exploratorios (AFE) para cada uno de Ios cuestionarios, y Análisis Factoriales Confirmatorios mediante el programa AMOS 18.

Para el estudio de diferencias, globales, por factores, y en función del género, edad, curso, tipo de centro y asignatura se utilizaron las pruebas ANOVA.

\section{Resultados}

En la Tablas 1, 2, 3 y 4 se presenta el alfa de Cronbach del cuestionario Cultura percibida y deseada del alumnado..

Tabla 1. Índice de fiabilidad alfa de Cronbach del cuestionario Cultura emprendedora percibida alumnado.

\begin{tabular}{cc} 
N de elementos & Alfa de Cronbach \\
\hline 24 & .916 \\
\hline
\end{tabular}

Tabla 2. Índice de fiabilidad alfa de Cronbach de los factores del cuestionario Cultura emprendedora percibida alumnado.

\begin{tabular}{lll}
\hline & N de elementos & Alfa de Cronbach \\
\hline Finalidad educativa & 6 & .816 \\
Proceso de Enseñanza & 6 & .732 \\
Proceso de Aprendizaje & 6 & .746 \\
Escenario & 6 & .630 \\
\hline
\end{tabular}




\section{INSTRUMENTOS DE EVALUACIÓN DE LA CULTURA EMPRENDEDORA}

Tabla 3. Índice de fiabilidad alfa de Cronbach del cuestionario Cultura emprendedora deseada alumnado.

\begin{tabular}{cc}
$\mathrm{N}$ de elementos & Alfa de Cronbach \\
\hline 24 & .888 \\
\hline
\end{tabular}

Tabla 4. Índice de fiabilidad alfa de Cronbach de los factores del cuestionario Cultura emprendedora deseada alumnado.

\begin{tabular}{lll}
\hline & N de elementos & Alfa de Cronbach \\
\hline Finalidad educativa & 6 & .735 \\
Proceso de Enseñanza & 6 & .652 \\
Proceso de Aprendizaje & 6 & .666 \\
Escenario & 6 & .757 \\
\hline
\end{tabular}

En las tablas 5.6.7.y 8 se presenta el alfa de Cronbach del cuestionario Cultura emprendedora percibida y deseada del profesorado.

Tabla 5. Índice de fiabilidad alfa de Cronbach del cuestionario Cultura emprendedora percibida profesorado

$\mathrm{N}$ de elementos Alfa de Cronbach

$24 \quad .887$

Tabla 6. Índice de fiabilidad alfa de Cronbach de los factores del cuestionario Cultura emprendedora percibida profesorado

\begin{tabular}{lll} 
& N de elementos & Alfa de Cronbach \\
\hline Finalidad educativa & 6 & .777 \\
Proceso de Enseñanza & 6 & .689 \\
Proceso de Aprendizaje & 6 & .717 \\
Escenario & 6 & .560 \\
\hline
\end{tabular}

Tabla 7.Índice de fiabilidad alfa de Cronbach del cuestionario Cultura emprendedora deseada profesorado

\begin{tabular}{cc} 
N de elementos & Alfa de Cronbach \\
\hline 24 & .919 \\
\hline
\end{tabular}

Tabla 8. Índice de fiabilidad alfa de Cronbach de los factores del cuestionario Cultura emprendedora deseada profesorado

\begin{tabular}{lll}
\hline & N de elementos & Alfa de Cronbach \\
\hline Finalidad educativa & 6 & .796 \\
Proceso de Enseñanza & 6 & .784 \\
Proceso de Aprendizaje & 6 & .757 \\
Escenario & 6 & .620 \\
\hline
\end{tabular}


A través del programa FACTOR 9.3.1, se realiza un análisis factorial de naturaleza exploratoria de la estructura factorial de los cuestionarios. No obstante, antes de llevar a cabo el análisis factorial, se emplearon los estadísticos de Kaiser-Meyer-Olkin (KM0) propuesto por Kaiser (1970) y la prueba de esfericidad de Bartlett, propuesta por Bartlett (1950); para comprobar la adecuación de los datos a este tipo de análisis.

En la Tablas 9, 19, 11 y 12, se presentan los datos de adecuación muestral: índice KaiserMeyer- Olkin y la prueba de Bartlett.

Tabla 9. Adecuación de la matriz de correlación: KMO, prueba de Bartlett y proporción de la varianza acumulada en cultura emprendedora percibida alumnado

\begin{tabular}{lcc}
\hline Factores & $\begin{array}{c}\text { Prueba de Esfericidad de } \\
\text { Bartlett }\end{array}$ & $\begin{array}{c}\text { Índice Kaiser- } \\
\text { Meyer-Olkin } \\
(\text { KMO) }\end{array}$ \\
\hline Finalidad & $4131.6(d f=15 ; p=.000)$ & .865 \\
Proceso de Enseñanza & $2289.8(d f=15 ; p=.000)$ & .819 \\
Proceso de Aprendizaje & $2451.3(d f=15 ; p=.000)$ & .830 \\
Escenario & $1598.7(d f=15 ; p=.000)$ & .700 \\
\hline
\end{tabular}

Tabla 10. Adecuación de la matriz de correlación: KMO, prueba de Bartlett y proporción de la varianza acumulada, Cultura emprendedora deseada alumnado

\begin{tabular}{lcc}
\hline Factores & $\begin{array}{c}\text { Prueba de Esfericidad de } \\
\text { Bartlett }\end{array}$ & $\begin{array}{c}\text { Índice Kaiser- } \\
\text { Meyer-Olkin } \\
(\text { KMO) }\end{array}$ \\
\hline Finalidad & $2470.5(d f=15 ; p=.000)$ & .813 \\
Proceso de Enseñanza & $1545.2(d f=15 ; p=.000)$ & .747 \\
Proceso de Aprendizaje & $1642.8(d f=15 ; p=.000)$ & .776 \\
Escenario & $1734.9(d f=15 ; p=.000)$ & .733 \\
\hline
\end{tabular}

Tabla 11. Adecuación de la matriz de correlación: KMO, prueba de Bartlett y proporción de la varianza acumulada, Cultura emprendedora percibida profesorado

\begin{tabular}{lcc}
\hline Factores & $\begin{array}{c}\text { Prueba de Esfericidad de } \\
\text { Bartlett }\end{array}$ & $\begin{array}{c}\text { Índice Kaiser- } \\
\text { Meyer-Olkin } \\
\text { (KMO) }\end{array}$ \\
\hline Finalidad & $266.9(d f=15 ; p=.000)$ & .792 \\
Proceso de Enseñanza & $160.7(d f=15 ; p=.000)$ & .707 \\
Proceso de Aprendizaje & $187.4(d f=15 ; p=.000)$ & .752 \\
Escenario & $141.1(d f=15 ; p=.000)$ & .595 \\
\hline
\end{tabular}




\section{INSTRUMENTOS DE EVALUACIÓN DE LA CULTURA EMPRENDEDORA}

Tabla 12. Adecuación de la matriz de correlación: KMO, prueba de Bartlett y proporción de la varianza acumulada, Cultura emprendedora deseada profesorado

\begin{tabular}{lcc}
\hline Factores & $\begin{array}{c}\text { Prueba de Esfericidad de } \\
\text { Bartlett }\end{array}$ & $\begin{array}{c}\text { Índice Kaiser- } \\
\text { Meyer-Olkin } \\
\text { (KMO) }\end{array}$ \\
\hline Finalidad & $312.9(d f=15 ; p=.000)$ & .822 \\
Proceso de Enseñanza & $298.6(d f=15 ; p=.000)$ & .772 \\
Proceso de Aprendizaje & $229.8(d f=15 ; p=.000)$ & .800 \\
Escenario & $169.7(d f=15 ; p=.000)$ & .744 \\
\hline
\end{tabular}

Para realizar los análisis del AFC se ha empleado el programa AMOS 18.0. Para el cálculo de los parámetros que compone el modelo, se utilizó como método de estimación Máxima Verosimilitud (Bollen, 1989).

En esta investigación han sido tenidos en cuenta los criterios de interpretación propuestos por Moral, Sánchez y Villarreal (2010). En la tabla 69 se observa, los índices de ajuste.

Tabla 13. Índices de ajuste

\begin{tabular}{|c|c|c|c|c|c|c|c|}
\hline & & & $\chi 2 \mathrm{ML} / \mathrm{gl}$. & $\mathrm{P}$ & SRMR & GFI & CFI \\
\hline \multirow{2}{*}{\multicolumn{2}{|c|}{ Criterios Moral et al. (2010) }} & Malo & $>3$ & $<.01$ & $>.099$ & $<.85$ & $<.85$ \\
\hline & & Bueno & $\leq 2$ & $\geq .05$ & $\leq .05$ & $\geq .95$ & $\geq .95$ \\
\hline \multirow{4}{*}{ Percibida } & \multirow{2}{*}{ Alumnado } & $\begin{array}{l}\text { Modelo } \\
1\end{array}$ & 7,062 & 0 & 0,0349 & 0,933 & 0,918 \\
\hline & & $\begin{array}{l}\text { Modelo } \\
2\end{array}$ & 1,305 & 0,271 & 0,003 & 0,999 & 1 \\
\hline & \multirow{2}{*}{ Profesorado } & $\begin{array}{l}\text { Modelo } \\
1\end{array}$ & 2,512 & 0 & 0,0834 & 0,761 & 0,725 \\
\hline & & $\begin{array}{l}\text { Modelo } \\
2\end{array}$ & 7,946 & 0 & 0,0376 & 0,959 & 0,961 \\
\hline \multirow{4}{*}{ Deseada } & \multirow{2}{*}{ Alumnado } & $\begin{array}{l}\text { Modelo } \\
1\end{array}$ & 8,942 & 0 & 0,0464 & 0,915 & 0,856 \\
\hline & & $\begin{array}{l}\text { Modelo } \\
2\end{array}$ & 18,911 & 0 & 0,0146 & 0,992 & 0,992 \\
\hline & \multirow{2}{*}{ Profesorado } & $\begin{array}{l}\text { Modelo } \\
1\end{array}$ & 2,861 & 0 & 0,0762 & 0,739 & 0,768 \\
\hline & & $\begin{array}{l}\text { Modelo } \\
2\end{array}$ & 4,213 & 0,015 & 0,0188 & 0,975 & 0,988 \\
\hline
\end{tabular}

En las Tablas 14, 15, 16 y17 se presentan los baremos para los cuatro cuestionarios elaborados en puntuaciones centiles. 
PSICOLOGÍA POSITIVA

Tabla 14. Baremo cultura emprendedora percibida alumnado

\begin{tabular}{lcccccc}
\hline & $\begin{array}{c}\text { Finalidad } \\
\text { educativa }\end{array}$ & $\begin{array}{c}\text { Proceso de } \\
\text { enseñanza }\end{array}$ & $\begin{array}{c}\text { Proceso de } \\
\text { aprendizaje }\end{array}$ & Escenario & General \\
\hline Media & 18,71 & 18,90 & 20,29 & 19,70 & 77,62 \\
Desv. Típ. & 4,70 & 4,17 & 4,33 & 4,213 & 15,50 \\
Mínimo & & 6,00 & 6,00 & 6,00 & 6,00 & 24,00 \\
Máximo & & 30,00 & 30,00 & 30,00 & 30,00 & 120,00 \\
Percentiles & 10 & 12,00 & 13,00 & 15,00 & 14,00 & 58,00 \\
& 20 & 15,00 & 15,00 & 17,00 & 16,00 & 65,00 \\
& 25 & 16,00 & 16,00 & 18,00 & 17,00 & 68,00 \\
& 30 & 16,00 & 17,00 & 18,00 & 18,00 & 70,00 \\
& 40 & 17,00 & 18,00 & 19,00 & 19,00 & 74,00 \\
& 50 & 19,00 & 19,00 & 20,00 & 20,00 & 78,00 \\
& 60 & 20,00 & 20,00 & 22,00 & 21,00 & 82,00 \\
& 70 & 21,00 & 21,00 & 23,00 & 22,00 & 86,00 \\
& 75 & 22,00 & 22,00 & 23,00 & 23,00 & 89,00 \\
& 80 & 23,00 & 22,00 & 24,00 & 23,00 & 91,00 \\
& 90 & 25,00 & 24,00 & 26,00 & 25,00 & 98,00 \\
\hline
\end{tabular}

Tabla 15. Baremo cultura emprendedora deseada alumnado

\begin{tabular}{|c|c|c|c|c|c|c|}
\hline & & Finalidad & $\begin{array}{l}\text { Proceso de } \\
\text { enseñanza }\end{array}$ & $\begin{array}{l}\text { Proceso de } \\
\text { aprendizaje }\end{array}$ & Escenario & General \\
\hline Media & & 24,89 & 24,70 & 24,95 & 25,89 & 100,45 \\
\hline Desv. Típ. & & 3,84 & 3,57 & 3,62 & 3,53 & 12,44 \\
\hline Mínimo & & 6,00 & 6,00 & 8,00 & 8,00 & 37,00 \\
\hline Máximo & & 30,00 & 30,00 & 30,00 & 30,00 & 120,00 \\
\hline \multirow[t]{11}{*}{ Percentiles } & 10 & 20,00 & 20,00 & 20,00 & 21,00 & 84,00 \\
\hline & 20 & 22,00 & 22,00 & 22,00 & 23,00 & 91,00 \\
\hline & 25 & 23,00 & 23,00 & 23,00 & 24,00 & 94,00 \\
\hline & 30 & 23,00 & 23,00 & 23,00 & 25,00 & 96,00 \\
\hline & 40 & 25,00 & 24,00 & 24,00 & 26,00 & 99,00 \\
\hline & 50 & 26,00 & 25,00 & 26,00 & 27,00 & 102,00 \\
\hline & 60 & 26,00 & 26,00 & 26,00 & 27,00 & 105,00 \\
\hline & 70 & 27,00 & 27,00 & 27,00 & 28,00 & 108,00 \\
\hline & 75 & 28,00 & 27,00 & 28,00 & 29,00 & 109,00 \\
\hline & 80 & 28,00 & 28,00 & 28,00 & 29,00 & 111,00 \\
\hline & 90 & 30,00 & 29,00 & 29,00 & 30,00 & 115,00 \\
\hline
\end{tabular}


Tabla 16. Baremo cultura emprendedora percibida profesorado

\begin{tabular}{|c|c|c|c|c|c|c|}
\hline & & Finalidad & $\begin{array}{l}\text { Proceso de } \\
\text { enseñanza }\end{array}$ & $\begin{array}{l}\text { Proceso de } \\
\text { aprendizaje }\end{array}$ & Escenario & General \\
\hline Media & & 23,90 & 23,10 & 24,55 & 22,34 & 93,92 \\
\hline Desv. Típ. & & 3,53 & 3,29 & 3,37 & 3,40 & 11,48 \\
\hline Mínimo & & 14,00 & 11,00 & 14,00 & 12,00 & 54,00 \\
\hline Máximo & & 30,00 & 30,00 & 30,00 & 30,00 & 119,00 \\
\hline \multirow[t]{11}{*}{ Percentiles } & 10 & 19,00 & 19,70 & 20,00 & 18,00 & 80,40 \\
\hline & 20 & 21,00 & 20,00 & 22,00 & 20,00 & 84,00 \\
\hline & 25 & 21,00 & 21,00 & 23,00 & 20,00 & 87,00 \\
\hline & 30 & 22,00 & 22,00 & 23,00 & 21,00 & 88,00 \\
\hline & 40 & 23,00 & 22,00 & 24,00 & 22,00 & 91,00 \\
\hline & 50 & 24,00 & 23,00 & 25,00 & 23,00 & 94,00 \\
\hline & 60 & 25,00 & 24,00 & 26,00 & 23,00 & 98,00 \\
\hline & 70 & 26,00 & 25,00 & 27,00 & 24,00 & 100,90 \\
\hline & 75 & 27,00 & 25,00 & 27,00 & 24,75 & 102,00 \\
\hline & 80 & 28,00 & 26,00 & 28,00 & 25,00 & 104,00 \\
\hline & 90 & 28,30 & 27,30 & 29,00 & 27,00 & 108,00 \\
\hline
\end{tabular}

Tabla 17. Baremo cultura emprendedora deseada profesorado

\begin{tabular}{|c|c|c|c|c|c|c|}
\hline & & Finalidad & $\begin{array}{l}\text { Proceso de } \\
\text { enseñanza }\end{array}$ & $\begin{array}{l}\text { Proceso de } \\
\text { aprendizaje }\end{array}$ & Escenario & General \\
\hline Media & & 27,51 & 27,49 & 27,38 & 26,09 & 108,48 \\
\hline Desv. Típ. & & 2,94 & 2,83 & 2,85 & 3,07 & 10,56 \\
\hline Mínimo & & 17,00 & 15,00 & 16,00 & 15,00 & 67,00 \\
\hline Máximo & & 30,00 & 30,00 & 30,00 & 30,00 & 120,00 \\
\hline \multirow[t]{11}{*}{ Percentiles } & 10 & 24,00 & 23,00 & 24,00 & 22,00 & 93,70 \\
\hline & 20 & 25,00 & 25,00 & 25,00 & 24,00 & 101,40 \\
\hline & 25 & 26,00 & 26,00 & 26,00 & 24,00 & 104,00 \\
\hline & 30 & 26,00 & 27,00 & 26,00 & 25,00 & 106,00 \\
\hline & 40 & 28,00 & 28,00 & 28,00 & 26,00 & 108,00 \\
\hline & 50 & 28,50 & 28,00 & 28,00 & 26,00 & 110,50 \\
\hline & 60 & 29,00 & 29,00 & 29,00 & 27,00 & 114,00 \\
\hline & 70 & 30,00 & 30,00 & 29,00 & 28,00 & 116,00 \\
\hline & 75 & 30,00 & 30,00 & 30,00 & 28,75 & 117,00 \\
\hline & 80 & 30,00 & 30,00 & 30,00 & 29,00 & 118,00 \\
\hline & 90 & 30,00 & 30,00 & 30,00 & 30,00 & 120,00 \\
\hline
\end{tabular}

\section{DISCUSIÓN}

Los datos que se aportan indican la presencia de unos instrumentos de evaluación de la cultura emprendedora acordes al modelo que se postula formulado por sus autores Martín del Buey y Martín Palacio, con todas las garantías de fiabilidad, validez y baremación exigidos por la ciencia psicométrica.

En relación a la fiabilidad el índice Alfa de Cronbach (Cronbach. 1951), universalmente acepta- 
do en la investigación psicométricas es superior al .75 en los cuatro instrumentos: siendo el de cultura percibida en los alumnos de .916, la deseada de .888. Y en los profesores la percibida de 0887 y la deseada de 9.69 .

En relación a la validez de estructura se ha obtenido una estructura factorial afín a la planteada en torno a la presencia de cuatro dimensiones. Entrando al detalle nos encontramos que en todos Ios análisis factoriales exploratorios de la cultura emprendedora percibida y deseada por el alumnado y percibida y deseada por el profesorado se observa la adecuación de una estructura de un solo factor de las dimensiones Finalidad, Proceso de Enseñanza, Proceso de Aprendizaje y Escenario respectivamente acerca de la cultura emprendedora, teniendo en cuenta el criterio de Timmerman y Lorenzo-Seva (2011) aceptado científicamente. Y por otro lado, se confirma en los cuatro instrumentos el modelo cultura que se compone de cuatro factores: Finalidad educativa, Proceso de enseñanza, Proceso de aprendizaje y Escenario educativo siguiendo el Modelo 2 del análisis confirmatorio que relaciona la cultura organizacional emprendedora con los cuatro factores tanto en cultura percibida como en deseada.

\section{CONCLUSIONES}

La cultura percibida y deseada de los alumnos y profesores analizada en base al constructo 0 modelo de análisis planteada por sus autores dispone de unos instrumentos que reúnen todos los requisitos reclamados por la psicometría como son los de fiabilidad, validez y baremación.

\section{REFERENCIAS BIBLIOGRÁFICAS}

Antúnez, A. (2012). Actualización del constructo Cultuaula y elaboración de un cuestionario de aplicación en las enseñanzas medias. Tesina de licenciatura. Universidad de Oviedo

Arnaiz Garcia, A (2015) la cultura emprendedora en la educación secundaria. Tesis doctoral Universidad de ovIEDO

Arnaiz, A., Di Giusto, C. y Zamorano, M. (2014). Cultura organizaiconal en la Universidad de Burgos. Estudio diferencia por ramas educativas. Actas del Congreso Internacioanl Docencia Universitaria e Innovación. Tarragona.

Arnaiz, A., Pizarro, J.P. y Martín del Buey, F. (2012). Análisis de la cultura organizacional percibida y paradigma por alumnos en contextos universitarios: estudio piloto de la facultad de psicología de la Universidad de Oviedo. Actas de VII congreso Iberoamericano de Docencia Universitaria. Faculdade de Psicologia e de Ciências da Educação da Universidade do Porto, Porto, Portugal.

Bartlett, M.S. (1950). Tests of significance in factor analysis. British Journal of Mathematical and Statistical Psychology, 3, 77-85.

Bonavia, T., Prado, V.J. y Barberá, D. (2009). Adaptación al castellano y estructura factorial del Denison Organizational Culture Survey. Psicothema, 21(4), 633-638.

Cronbach, L.J. (1951). Coefficient alpha and the internal structure of tests. Psychometrika, 16, 297334.

De la Torre Cruz, T., Ruiz Palomo, E. Escolar Llamazares, M.C., Baños Martinez,V., Gañan Ibañez, A. , Corbi Santamaria, M., Santos Gonzalez, J y Sanchez, I (2015) : Impacto de la educación en la cultura del emprendimiento: estudio cualitativo. International. Journal of Developmental and Educational Psychology. Infad Revista de Psicología, n 1, Vol,2 , pp 117-128

Denison, D.R., y Neale, W. (1994). Denison Organizacional Culture Survey. Ann Arbor: Aviat.

Denison, D.R., y Neale, W. (2000). Denison Organizational Culture Survey. Ann Arbor: Denison Consulting

Di Giusto, C., Castellanos, S. y Arnaiz, A. (2014). Cultura organiazcional en la Universidad de Burgos. 


\section{INSTRUMENTOS DE EVALUACIÓN DE LA CULTURA EMPRENDEDORA}

Estudio diferenciado por género. Actas del Congreso Internacioanl Docencia Universitaria e Innovación. Tarragona.

Grady, N.B., Fisher, D.L. y Fraser, B.J. (1996). Images of school through metaphor development and validation of a questionnaire. Journal of Educational Administration, 34(2), 41-53.

Harrison, R. y Stokes, H. (1992). Diagnosing Organizational Culture. Amsterdam: Pfeiffer \& Company

Houtveen, A.A.M., Voogt, J.C., Van der Vegt, A.L. y Van de Grift, W.J.C.M. (1996). Zo zijn onze manieren: onderzoek naar de organisatiecultuur van scholen. Utrecht: Universiteit Utrecht.

Jones, R. (1996). The school culture inventory: a tool for identifying values, setting goals and bringing about school improvement. Education Canada, 36(4), 6-10.

Kaiser, H.F. (1970). A second generation little jiffy. Psychometrika, 35, 401-415.

Luis Rico, M.I., Palmero Cámara, C., De la Torre Cruz, T., Ruiz Palomo, E., Corbi Santamaria, M. Sanchez M.I., Jimenez Eguizabal, A. (2015) Redes de emprendimiento: percepción y efectos de la cultura emprendedora. International Journal of Developmental and Educational Psychology. Infad Revista de Pscologia, n 1, Vol,2 , pp 183-196

Lorenzo-Seva, U. y Ferrando, P.J. (2013). FACTOR 9.2 A Comprehensive Program for Fitting Exploratory and Semiconfirmatory Factor Analysis and IRT Models. Applied Psychological Measurement, 37, 497-498.

Lorenzo-Seva, U. y Ferrando, P.J. (2015). Manual of the program Factor. Recuperado en marzo, 2015, disponible en: http://psico.fcep.urv.es/utilitats/factor/.

Marcone, R. y Martín del Buey, F. (2003). Construcción y validación de un inventario de cultura organizacional educativa (ICOE). Psicothema, 15(2), 292-299.

Martín del Buey, F. (1991). Modelo de análisis de presunciones culturales en el aula. Actas del I Congreso Internacional de Psicología y educación (pp. 168-170). Madrid.

Martín del Buey, F. (1997). Modelos implícitos en la reforma educativa. Revista Magister, 15, 90-97.

Martín del Buey, F. y Martín Palacio, M.E. (2012). Análisis de la cultura en el aula: CULTUAULA. Material inédito. Editado en formato digital sin finalidad venal.

Martín Palacio, M.E., Aguado, J.J. y Rodríguez, E. (2013). Cultura percibida y deseada en contextos educativos universitarios. En J.J. Gázquez Linares, M.C. Pérez Fuentes y M.M. Molero Jurado (Comps.), La Convivencia Escolar: Un acercamiento multidisciplinar (pp. 69-74). Almería: ASUNIVEP

Meza, C.L. (2010). Cultura escolar inclusiva en Educación Infantil: Percepciones de profesionales y padres. Tesis doctoral. Universidad de Salamanca.

Moral, J.C., Sánchez, J.C. y Villarreal, M.E. (2010). Desarrollo de una escala multidimensional breve de ajuste escolar. Revista Electrónica de Metodología Aplicada, 15(1), 1-11.

Pang, N.S.K. (1995). The development of the school values inventory. En R. Cotter, y S.J. Marshall (Eds), Research and practice in educational administration (pp. 160-86). Victoria: Australian Council for Education Administration

Reyes, M. y Zambrano, V. (1991). Un instrumento para medir la cultura organizacional innovadora. Tesis doctoral. Santiago de Chile Chile: Universidad Central.

Sashkin, M. y Sashkin, M.G. (1990). Leadership and culture-building in schools: Quantitative and qualitative understandings. Paper presented at the annual meeting of the American Education Research Association, Boston, MA.

Sanchez, J.C., Caggiano, V. Hernández, B. (2011): Competencias emprendedoras en la educación universitaria International Journal of Developmental and Educational Psychology. Infad Revista de Pscologia, n 1, Vol,3 , pp 19-28 